\title{
A GENERALIZATION OF AN INEQUALITY OF COPPEL
}

\author{
G. A. HEWER
}

ABSTRACT. Upper and lower bounds for the solutions of a linear ordinary differential equation are determined from the solutions of upper and lower matrix comparison equations. The coefficients of the comparison equations are computed with the help of Lozinskiî's logarithmic "norm"

$$
l(A)=\lim _{h \rightarrow+0}[|I+h A|-1] / h,
$$

and the concept of the "matricial norm" as a matrix of scalar norms. Using these estimates some new criteria for the stability of composite systems are obtained.

1. Introduction. The Lozinskiĭ logarithmic norm [8] of an $n \times n$ matrix $A$ is

$$
l(A)=\lim _{h \rightarrow+0}(|I+h A|-1) / h
$$

where $|\cdot|$ is any matrix norm compatible with some vector norm $\eta(\cdot)$. The purpose of this note is to introduce a matricial logarithmic norm (a mapping from a set of square matrices into a set of equal or lower dimensional matrices) and thereby obtain new bounds for the solutions of ordinary linear differential equations. These new bounds subsume those estimates obtained in the following theorem by W. A. Coppel [2, p. 48].

THEOREM 1. If the $n \times n$ matrix $A(t)$ is defined and continuous for $t \geqq t_{0} \geqq 0$, then every solution of the equation

$$
\dot{x}=A(t) x,
$$

satisfies the estimate

$$
\eta\left(x\left(t_{0}\right)\right) \exp \left(-\int_{t_{0}}^{t} l(-A(s)) d s\right) \leqq \eta(x(t)) \leqq \eta\left(x\left(t_{0}\right)\right) \exp \int_{t_{0}}^{t} l(A(s)) d s
$$
for $t \geqq t_{0} \geqq 0$.

Received by the editors July 18, 1973 and, in revised form, August 30, 1973.

AMS (MOS) subject classifications (1970). Primary 34A40.

Key words and phrases. Differential inequalities, positive matrix, matricial and vectorial norms, logarithmic norm.

(c) American Mathematical Society 1974 
In other words, upper and lower bounds for any solution of (1.1) are determined by the first order comparison equation $\dot{y}=l(A(t)) y$. For example, if $\eta(x)=\left(\sum_{i=1}^{N}\left|x_{i}\right|^{2}\right)^{1 / 2}$ (Euclidean norm), then $l(A)$ is the largest eigenvalue of $\frac{1}{2}\left(A+A^{\prime}\right)\left(A^{\prime}\right.$ being the transpose of $\left.A\right)$. With this norm the estimate (1.2) is attributed to Wintner [12] or Wazewski [11]. Other generalizations of this inequality are obtained in [6, p. 153] or [9] by extending the definition of $l(\cdot)$ to include Banach space operators.

In all cases the domain of the function $\exp (\cdot)$ in inequality (1.2) is determined by a real valued function $l(\cdot)$. Here an estimate for the solutions of (1.1) is obtained in which the domain of the function $\exp (\cdot)$ is a set of $s \times s$ matrices $(1 \leqq s \leqq$ order of $A$ ). This new estimate provides upper and lower bounds for solutions of (1.1) as determined by an $s$ th order linear differential comparison equation whose fundamental matrix solution is a positive matrix. Not only does this subsume Coppel's estimate, but the stability behavior of (1.1) can be determined from a matrix function of $A$. This feature should be useful when the dimension of $A$ is large and $A$ can be partitioned into subblocks whose stability behavior can be easily determined. Finally, as an application of this estimate, some new stability criteria for partitioned systems are given in terms of this matrix function of $A$.

2. Matricial logarithmic norms. First we recall some terminology and introduce some notation.

Let $R^{n}$ denote a real $n$-dimensional vector space with natural partial order defined between any two vectors $x, y \in R^{n}$ by $x \leqq y$ if and only if $x_{i} \leqq y_{i}(i=1, \cdots, n)$ and $R_{+}^{n}$ be the set of $x \in R^{n}$ with $x \geqq 0$. The set $\mathscr{P}_{s}=\left\{P_{1}, \cdots, P_{s}\right\}$ of $n \times n$ projection matrices which satisfy the relations $P_{s}^{2}=P_{s}, P_{i} P_{j}=0=$ the null matrix $i \neq j$ and $P_{1}+\cdots+P_{s}=I=$ the identity matrix, is a partition set for $A$ whenever its elements commute with $A$.

The vector valued function $N_{s}(x)=\left(\eta\left(P_{1} x\right), \cdots, \eta\left(P_{s} x\right)\right)$ is a mapping from $R^{n}$ into $R_{+}^{s}$ and satisfies the three conditions

(i) $N_{s}(x)=|\lambda| N_{s}(x)$ ( $\lambda$ is any real number),

(ii) $N_{s}(x+y) \leqq N_{s}(x)+N_{s}(y)$,

(iii) $N_{s}(x)=0$ implies $x=0$ in $R^{n}$,

which are the axioms of a vectorial norm [10, p. 267]. For any $n \times n$ matrix $A$ the $s \times s$ matrix

$$
M_{s}(A)=\left(\left|P_{i} A P_{j}\right|\right)_{i, j=1, \ldots, s}
$$

is a mapping from the set of $n \times n$ matrices with entries from $R$ into the set of $s \times s$ matrices with entries from $R_{+}$and is a matricial norm as defined in [3] or [10]. This norm satisfies (with obvious modification) properties (i), (ii), and (iii) of the vectorial norm $N_{s}(\cdot)$. 
Clearly, when $\mathscr{P}_{1}=\{I\}$ (the identity partition), then $N(x)=\eta(x)$ and $M_{1}(A)=|A|$.

PROPOSITION 1. If $|\cdot|$ and $\eta(\cdot)$ are compatible, then so are $N_{s}(\cdot)$ and $M_{s}(\cdot)$ compatible, i.e., $N_{s}(A x) \leqq M_{s}(A) N_{s}(x)$ for any $n \times n$ matrix $A$ and $1 \leqq s \leqq n$.

Proof. For any partition set $\mathscr{P}_{s}$, using the definition of a partition set, we have

$$
\begin{aligned}
N_{s}(A x) & =\left(\eta\left(P_{1} A x\right), \cdots, \eta\left(P_{s} A x\right)\right) \\
& =\left(\eta\left(P_{1} A\left(\sum_{i=1}^{s} P_{i}\right) P_{1} x\right), \cdots ; \eta\left(P_{s} A\left(\sum_{i=1}^{s} P_{i}\right) P_{s} x\right)\right) \\
& \leqq M_{s}(A) N_{s}(x) .
\end{aligned}
$$

The partial order $B \leqq A$ for any two square matrices means that $x^{\prime}(A-B) x \geqq 0$.

The matricial logarithmic norm for any $n \times n$ matrix $A$ and partition set $\mathscr{P}_{s}$ is the $s \times s$ matrix

$$
L_{s}(A)=\lim _{h \rightarrow+0}\left(M_{s}(I+h A)-M_{s}(I)\right) / h .
$$

For completeness we show that the limit in (2.1) exists. For any $0<\theta<1$, $M_{s}(I+\theta h A) \leqq \theta M_{s}(I+h A)+(1-\theta) M_{s}(I)$ and hence

$$
(\theta h)^{-1}\left(M_{s}(I+\theta h A)-M_{s}(I)\right) \leqq h^{-1}\left(M_{s}(I+h A)-M_{s}(I)\right) \leqq M_{s}(A) .
$$

Thus, the difference ratio in (2.1) is a nondecreasing function of $h$ and is bounded by the matrix $\pm M_{s}(A)$.

As an example, if $\eta(x)$ is the Euclidean norm, then the diagonal elements of $L_{s}(A)$ will be the largest eigenvalues of the main diagonal partitions of $A$ and the off-diagonal elements of $L_{s}(A)$ will be the spectral norm of the matrices in the off-diagonal partitions of $A$. Also, for the identity partition $L_{1}(A)=l(A)$.

Just as $l(A)$ which can assume negative values is not a matrix norm, $L_{s}(A)$ is not a matricial norm. However, they do have the following properties in common.

THEOREM 2. If $A$ and $B$ are any $n \times n$ matrices and $\mathscr{P}_{s}$ is any partition set for $A$ and $B(1 \leqq s \leqq n)$, then

(a) $L_{s}(\lambda A)=|\lambda| L_{s}(A)$ ( $\lambda$ any real number $)$,

(b) $L_{s}(A)=L_{s}\left(A^{\prime}\right)$,

(c) $L_{s}(A+B) \leqq L_{s}(A)+L_{s}(B)$,

(d) $M_{s}\left(L_{s}(A)\right) \leqq M_{s}(A)$,

(e) $M_{s}\left(L_{s}(A)-L_{s}(B)\right) \leqq M_{s}(A-B)$,

(f) $M_{s}(\exp A) \leqq \exp L_{s}(A)$. 
Proof. Properties (a), (b), and (c) are easily verified and (d) follows because as already shown $-M_{s}(A) \leqq L_{s}(A) \leqq M_{s}(A)$. Property (e) is a consequence of (c) and (d).

Finally to prove (f), we merely sketch the proof. For every $\varepsilon>0$ there exists a positive integer $m_{0}$ such that for $m \geqq m_{0}, M_{s}(\exp A)-$ $M_{s}\left((I+A / m)^{m}\right) \leqq \varepsilon M_{s}(1)\left(M_{s}(1)\right.$ has all entries equal to 1$)$. Whence,

$$
\begin{aligned}
& M_{s}(\exp A) \\
& \quad \leqq M_{s}\left((I+A / m)^{m}\right)+\varepsilon M_{s}(1) \\
& \quad \leqq\left(M_{s}(I)+m\left(M_{s}(I+A / m)-M_{s}(I)\right) / m\right)^{m}+\varepsilon M_{s}(1) \\
& \quad \leqq\left(M_{s}(I)+\left(L_{s}(A)+\varepsilon M(1)\right) / m\right)^{m}+\varepsilon M_{s}(1) \rightarrow \exp \left(L_{s}(A)\right) \quad \text { as } m \rightarrow \infty .
\end{aligned}
$$

3. A generalization of Coppel's inequality. Let $Y(t)$ denote the fundamental matrix solution of the comparison equation for $t \geqq t_{0} \geqq 0$,

$$
\dot{Y}=L_{s}(A(t)) Y, \quad Y\left(t_{0}\right)=I .
$$

Since the off-diagonal elements of the coefficient matrix in (3.1) are all nonnegative, $Y(t) \geqq 0\left(Y(t)\right.$ is a nonnegative matrix) for $t \geqq t_{0} \geqq 0$ [1, p. 138].

THEOREM 3. If $\mathscr{P}_{s}$ is any partition set for $A(t)$, then every solution of (1.1) satisfies the estimate for $t \geqq t_{0} \geqq 0$

$$
Y(-t) N_{s}\left(x\left(t_{0}\right)\right) \leqq N_{s}(x(t)) \leqq Y(t) N_{s}\left(x\left(t_{0}\right)\right) .
$$

Proof. Let $r(t)=N_{s}(x(t))$ and

$$
\dot{r}_{+}(t)=\lim _{h \rightarrow+0}\left(N_{s}(x(t)+h \dot{x}(t))-N_{s}(x(t))\right) / h .
$$

Properties (i) and (ii) of a vectorial norm are sufficient [2, p. 3] to show that this limit exists. Using Proposition 1, we have

$$
N_{s}(x(t)+h \dot{x}(t))=N_{s}((I+h A(t)) x(t)) \leqq M_{s}(I+h A(t)) N_{s}(x(t)),
$$

whence $\dot{r}_{+}(t) \leqq L_{s}(A(t)) r(t)$. The vector $Y(t) N_{s}\left(x\left(t_{0}\right)\right)$ is a solution of (3.1) and is an upper bound for the latter differential inequality $[6$, p. 28] and so the first half of the inequality is established. The other half of the theorem is obtained by replacing $t$ by $-t$. Clearly, for the identity partition Coppel's inequality is obtained.

4. Diagonally dominant matrices, positive matrices and stability. The stability behavior of (1.1) can often be inferred from the corresponding behavior of (3.1). Such techniques are illustrated in [6]. In this section, we assume that $A$ is a constant matrix and obtain some stability criteria for (1.1) which exploit the matrix properties of $L_{s}(A)$ and $\exp \left(L_{s}(A)\right)$. 
The matrix $L_{s}(A)$ is diagonally dominant if

$$
l\left(P_{j} A P_{j}\right) \leqq-\sum_{k=1 ; k \neq j}^{s}\left|P_{j} A P_{k}\right| \quad(j=1, \cdots, s),
$$

THEOREM 4. Given some partition set $\mathscr{P}_{s}$ of $A$, if the matrix $L_{s}(A)$ is diagonally dominant or the determinants of the nested set of principal minors of $L_{s}(A)$ satisfy the inequalities

$$
\begin{aligned}
& l\left(P_{1} A P_{1}\right) \leqq 0, \quad \operatorname{det}\left(\begin{array}{cc}
l\left(P_{1} A P_{1}\right) & \left|P_{1} A P_{2}\right| \\
\left|P_{2} A P_{1}\right| & l\left(P_{2} A P_{2}\right)
\end{array}\right) \geqq 0, \cdots,
\end{aligned}
$$

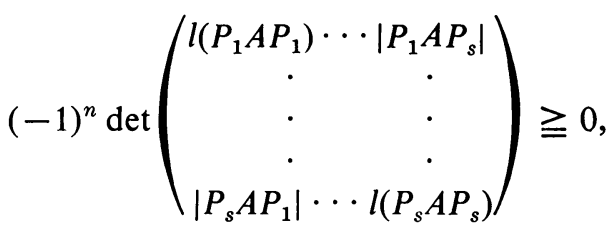

and $\left|P_{i} A P_{j}\right|$ is nonzero for $i \neq j$, then every solution of (1.1) is uniformly stable. If the inequalities in (4.1), (4.2) are all strict, then every solution of (1.1) is asymptotically stable.

Proof. By Geršgorin's theorem [7] the real parts of the eigenvalues of a (strictly) diagonally dominant matrix are all (less than) zero or less than or equal to zero. The inequalities in (4.2) assure the same eigenvalue behavior $[4, \mathrm{p}$. 74]. If the inequalities are all strict, then the real parts of the eigenvalues of the fundamental matrix solution $\exp L_{s}(A) t$ of (3.1) are all less than one and the proof is completed.

On the other hand, suppose they are not all strict and 1 is an eigenvalue of the positive matrix solution [1, p. 137]. By Perron's theorem [7, p. 286], 1 is a simple eigenvalue and is greater than the modulus of any other eigenvalue of this matrix. As is well known, this is sufficient for this matrix to be uniformly bounded, which completes the proof.

In view of these results, other stability and instability criteria could also be stated. For example, whenever $P_{s}$ is the identity partition, any stability criteria involving inequalities (4.2) include those of Coppel [2, p. 49]. Finally, by either using the composite estimate $I\left(L_{s}(A)\right)$ or $L_{s}(A)$ directly other stability criteria could be obtained.

\section{REFERENCES}

1. E. F. Beckenbach and R. Bellman, Inequalities, Ergebnisse der Math. und ihrer Grenzgebiete, Band 30, Springer-Verlag, Berlin, 1961. MR 28 \#1266.

2. W. A. Coppel, Stability and asymptotic behatior of differential equations, D. C. Heath, Boston, Mass., 1965. MR 32 \#7875. 
3. E. Deutsch, Matricial norms, Numer. Math. 16 (1970/71), 73-84. MR 43 \#3285.

4. F. R. Gantmaher, The theory of matrices, GITTL, Moscow, 1953; English transl., Vol. 2, Chelsea, New York, 1959. MR 16, 438; 21 \#6372c.

5. G. E. Ladus and V. Lakshmikantham, Differential equations in abstract spaces, Academic Press, New York, 1972.

6. V. Lakshmikantham and S. Leela, Differential and integral inequalities. Vol. 1, Academic Press, New York, 1969.

7. P. Lancaster, Theory of matrices, Academic Press, New York, 1969. MR 39 \#6885.

8. S. M. Lozinskiī, Error estimates for the numerical integration of ordinary differential equations. I, Izv. Vysš. Učebn. Zaved Matematika 1958, no. 5 (6), 52-90; errata, 1959, no. 5 (12), 222. MR 26 \#3191.

9. R. H. Martin, Jr., Bounds for solutions of a class of nonlinear different ial equations, J. Differential Equations 8 (1970), 416-430. MR 44 \#1874.

10. F. Robert, Norms veciorielles cie üecteurs et de mütrices, Rev. Française Traitement Information Chiffres 7 (1964), 261-299. MR 34 \#5261.

11. T. Wazewski, Sur la limitation des intégrales des systèmes d'équations différentielles linéaires ordinaires, Studia Math. 10 (1948), 48-59. MR 10, 40.

12. A. Wintner, Asymptotic integration constants, Amer. J. Math. 68 (1946), 553559. MR 8, 272.

Michelson laboratories, Naval Weapons Center, China lake, California 93555 